\title{
Defect of Septum Atrio-Ventricular Complete in Newborn. Clinical Evolution Report of a Case
}

\begin{abstract}
Summary
Congenital heart disease has an incidence and prevalence ranging from $0.8 \%$ to $1 \%$, approximately $7.3 \times 1,000(0.73 \%)$ of live births, are the second cause of death in Mexico according to statistics from the National Institute of Statistics and Geography, mortality is related to the type of heart disease, gestational age, weight of the newborn, and the place where the patient is attended at birth. The complete atrio-ventricular septum defect is a rare condition of very high mortality in the first year of life. Its timely diagnosis prevents complications such as hypertension, heart failure and death. We present the clinical evolution of a 6 - month - old male with this heart disease, which, through surgery, separated the systemic and pulmonary circulation, performing anastomosis of the systemic venous return to the pulmonary circulation, presenting surgical complications associated with this congenital malformation.
\end{abstract}

Keywords: Defects of the interventricular septum; Congenital heart defects; Surgical complications

\section{Introduction}

Congenital heart disease is the major cause of congenital malformations at birth. According to different reports, the incidence and prevalence range from $0.8 \%$ to $1 \%$ of $7.3 \times 1,000$ $(0.73 \%)$ of live births [1]. The most frequent types of heart disease were prematurity of the ductus arteriosus and prematurity interauricular communication and in those of interventricular communication and interauricular communication, thirdly the persistence of ductus arteriosus [2,3]. Causes are unknown in large numbers. Inheritance is a determining factor in $8 \%$ of cases, teratogens in 1 to $2 \%$ and $90 \%$ in multifactorial cases. [4].

Congenital heart disease is the second leading cause of death in Mexico according to statistics from INEGI (National Institute of Statistics and Geography). Mortality is influenced by the place where the patient is treated and secondly by clinical manifestations at birth such as cyanosis complex heart disease with a less favorable prognosis than the rest of the heart diseases $[5,14]$.

\section{Case}

A 6-month-old male with a perinatal history of being a product of gestation 1 of a 19-year-old mother from Xalapa Veracruz, who was employed with prenatal control, enrolled in a persistent urinary tract infection after the third month of gestation, denied vaginal infections, threat of abortion, diabetes and hypertension obtained by eutococcal calving qualified with apgar of 8-9, weighing $3050 \mathrm{~kg}$, of 40 weeks of gestation, presented hypothermia in the first three hours of its birth later rejection to the pathway oral, hypoactivity, weak suction and hypoglycemia, peri-oral cyanosis with 55\% oxygen saturation by pulse oximetry, physical examination shows a systolic ejection murmur grade II of VI, second intense noise, hospitalized and assessed by pediatric cardiology performing echocardiogram finding single atrium,

Case Report
Volume 10 Issue 2 - 2017
Alicia Santa Cortes González*1, Jennifer
del rosario Molina Domínguez ${ }^{1}$ and Héctor
Hugo López Medina
${ }^{1}$ Universidad Veracruzana, Servicio de pediatría hospital
general de zona núm. 11 Instituto Mexicano del seguro social
de Xalapa Veracruz, México
${ }^{2}$ Universidad Veracruzana, Servició de Medicina Familiar
Unidad de Medicina Familiar núm. 19 Instituto Mexicano del
seguro social de Xalapa Veracruz, México
*Corresponding author: Alicia Santa Cortés González
Servicio de Pediatría HGZ No. 11 Mexican Social Insurance
Institute "Dr. Ignacio García Téllez “Lomas del Estadio S / N
Col. Centro, Xalapa Veracruz, Mexico Tel: 228 8 18-55-55;
Ext. 61383 0 61334; Email: aliciasantac@hotmail.com
Received: October 12, 2017 | Published: December 01,
2017

right single ventricle, with poor po pulmonary artery hypoplasia, unbalanced AV channel, which is sent to the third level of care for diagnostic complementation and specialized treatment, where echocardiography is performed again, reporting a double entry to the left ventricle, a common AV valve, right ventricle double outlet, poorly placed vessels, anterior and left aorta, infundibular and valvular mixed pulmonary stenosis with ring hypoplasia, confluent pulmonary branches, with right branch origin stenosis of $2.1 \mathrm{~mm}$, distal $3 \mathrm{~mm}$, left aortic arch without obstruction with pulse oximetry between 35 and $67 \%$ of oxygen saturation was managed with infusion of milrinone, epinephrine and furosemide for a few hours, right systemic pulmonary systemic fistula is performed through thoracotomy dissecting by planes to pleural cavity with dissection of the artery subclavian and right pulmonary artery, were real an anastomosis of gorotex to the right subclavian artery and posteriorly to the right pulmonary artery, during the procedure has significant saturation and decreased heart rate merits advanced resuscitation maneuvers for three minutes on two occasions, after the surgical procedure chest x-ray is performed which shows, levocardia, levoapex, decreased pulmonary flow, visceral heterotaxia, right diaphragmatic paralysis (Figure 1) a pleural tube was placed to reduce the diaphragmatic paralysis, with improvement of ventilation remained in mechanical ventilation for a week, presenting to the extubation again severe respiratory difficulty with over-distension left lung suspected airway injury bronchoscopy is performed being severe tracheoendobronquitis, laryngomalasia degree I, and erosion of 
the left vocal cord begins treatment with steroid and antibiotic by isolation of germ enterobacter cloacae multiresistent, performs tracheotomy and placement of cannula shiley 4.0 , is performed esophagogastroduodenal series to rule out anatomical alterations finding passage of the contrast medium a the trachea with alteration of the phase 2 of the swallowing mechanics with passage to the airway being made fundoplication with gastrostomy with progressive increase of the oral route. A few hours after the first surgical event and once suspended sedation presents subtle convulsive seizures characterized by blinking and sucking, and treatment with phenobarbital is performed. Tomography of the skull is found. Sequelae of hypoxic-ischemic encephalopathy and right parietal-temporal-occipital cerebral infarction were discharged in stable conditions at 57 days of age. Currently the patient is in the 3 th percentile low for his age with acute malnutrition without affectation of the size. With adequate age-specific blood pressure, gastrostomy and tracheotomy with pulse oximetry between 83 and $90 \%$, with a good evolution in pediatric and pediatric cardiology.

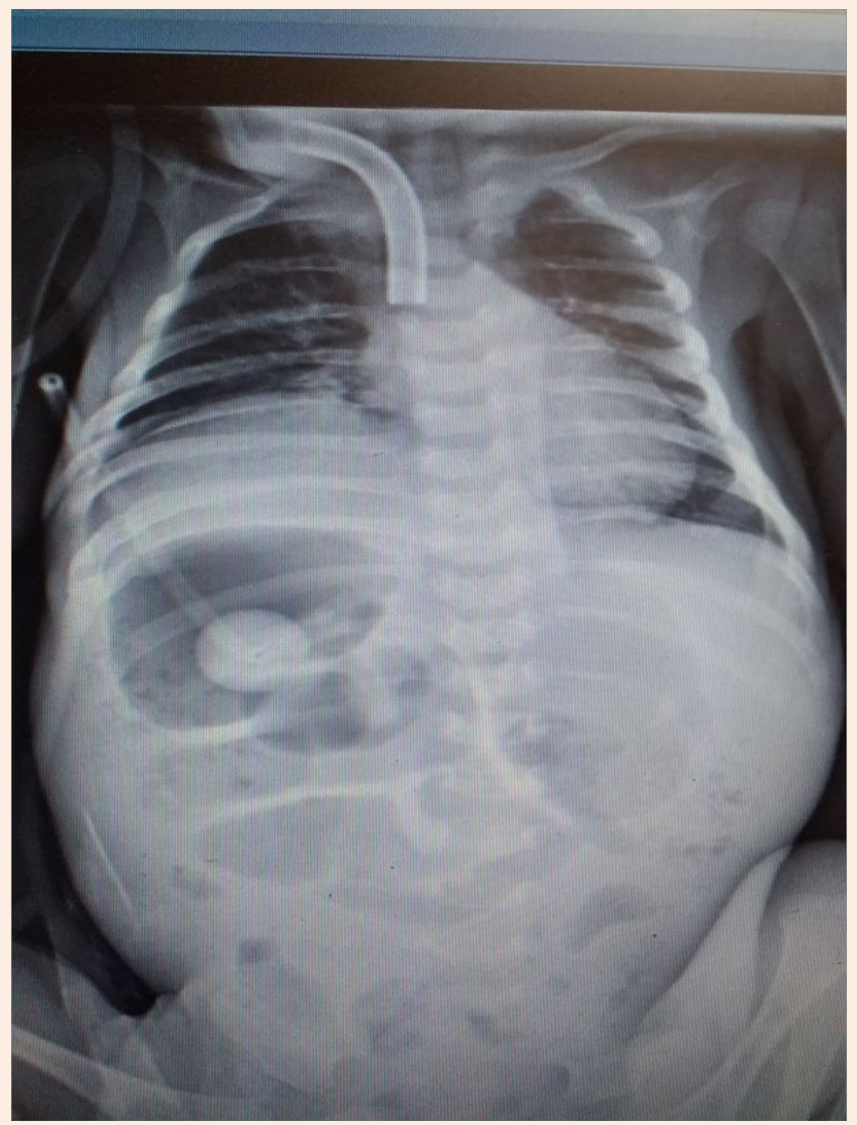

Figure 1: Chest X-ray showing right diaphragmatic paralysis.

\section{Discussion}

The complete atrioventricular canal defect involves both inferior and superior compartments, the tricuspid and mitral valves are not formed as individual valves. A single ventricle is formed as a rare pathology and with the possibility of surgical treatment by means of palliative surgeries, when it is diagnosed in $[6,7]$ the age and weight of the patient are determinants for their survival, as well as the early diagnosis of the anomaly, in order to avoid complications such as pulmonary hypertension, heart failure and death. that the patient can perform the surgery at an appropriate age, without it worsening his physical condition, for which he is supported with medication and continuous medical care [8-10]. In this patient, the symptoms that I present during the first hours of life such as cyanosis and difficulty feeding and the presence of heart murmur made suspicion In the case of complex heart disease, the hospital where this case was analyzed has no hemodynamic or cardiac surgery service, so it had to move urgently with life support and drugs to a third level for its specialized care.

Through surgery they separated the systemic and pulmonary circulation and anastomosis of the systemic venous return to the pulmonary circulation was performed. This surgery is usually achieved in two stages: connection of the superior vena cava to the pulmonary artery around 4-6 months of life (Glenn), to be completed later with connection of the inferior vena cava to the pulmonary circuit around the 4-6 years (Fontan), the quality of life of these patients is acceptably good, although in the long term (3-4 decades) complications can arise, they frequently require reoperations and eventually end up needing heart transplantation [11-13] According to comparative studies in the united states, the single ventricle manifests itself in 5 out of every 100,000 live births, the treatment is of the medical and surgical type which is more a palliative method than correction. With Fontan surgery, it is sought to close all communications between the right and left hemicardium and connect the cavas with the pulmonary arteries [15].

During the surgery, the patient presented significant desaturation of oxygen and bradycardia requiring advanced resuscitation maneuvers that conditions hypoxic ischemic encephalopathy, with frequent endotracheal intubations injuring the airway with bacterial superinfection by enterobacter cloacae and nosocomial sepsis by multiresistant klebsiella pneumoniae, with tracheotomy, with alterations in the mechanics of swallowing by cerebral hypoxia being meritorious of gastrostomy and treatment with anticonvulsivante for the development of secondary seizures.

\section{Conclusion}

The clinical evolution of this patient was determined by the timely detection of the complications presented during the performed surgeries, the clinical manifestations presented at his birth determined the immediate management of his cardiopathy presenting lesions with high surgical mortality during his treatment and high morbidity in the long term. In this work there is no conflict of interest or any kind of financing, informed consent was made in the patient's care.

\section{References}

1. Mendieta G, Santiago A, Mendieta Z. Incidence of congenital heart disease and factors associated with lethality in children born in two hospitals in the State of Mexico. Medical Gazette of Mexico 2013; 149: 617-623. 
2. Olivia J, Zeballos R, R. W. Ventriculo Congenita Cardiopathy. Gaceta Medica 2005; 47-51.

3. Achacollo H, Martinez H, Adauto J. Ventriculo Single in a 5-month patient, report of a case. Rev Cient Cienc Med 2010; 13 (2): 96-98

4. Alva C. Complete transposition of the great arteries. Medical Evidence And Research In Health 2013; 6 (2): 55-58

5. Quesada T, Maribel C. Congenital heart disease until the neonatal stage. Clinical and Epidemiological Aspects. Acta Medica del Centro 2014; 8 (3): 149-162.

6. Cárdenas L, Enriquez G, Haecker S. Newborn Carrier of Complex Congenital Heart Disease. Risk analysis, decision making and new therapeutic possibilities. Rev. Med. Clin. Condes 2016; 27 (4): 476 484.

7. Alfonso L, J. Approach of the Child with Suspected Congenital Heart Disease. Revista Mexicana de Pediatría 2015; 83 (3): 104-113.

8. Calderón J, Cervantes J, Curi P. Problems of congenital heart disease in Mexico. Proposal of Regionalization. Arch Cardiol Mex 2010; 80 (2), 133-140,

9. Dragulescu A, Kammache I, Fouilloux V. Long-term results of pulmonary artery rehabilitation in patients with pulmonary atresia, ventricular septal defect, pulmonary artery hypoplasia, and major aortopulmonary collaterals. The Journal of Thoracic Surgery 2011; 142 (6); 1374-1380.
10. Turón A, Riverola A, Moreno j. Characteristics and Outcomes of Transposition of Great Arteries in the Neonatal Period. Rev Esp Cardiol 2014; 62 (2): 114-119.

11. Clavaruso D, Ocello S, Salviato N. Implatation of a Berlin Heart as Single Ventricle By-Pass on Fontan Circulation in Univentricular Heart Failure. ASAIO Journal 2007; 1-2.

12. Hoffman Jl, Kaplan S. The incidence of congenital heart disease. J Am Coll Cardiol. 2002; 39 (12): 1890-900.

13. Guerchicoff M, Marantz P, Infante J. et al. Evaluation of the impact of the early diagnosis of congenital heart disease. Arch Argent Pediatr 2004; 102: 445-70.

14. National Institute of Statistics and Geography: Women and Men in Mexico 2010 (Monograph). Mexico. [Internet] Accessed October 05, 2017. Available at: http://www.inegi.org.mx/ prod_serv/contenidos/espanol/bvinegi/productos/integración/ sociodemografico/mujeresyhombres/2010/MyH_2010.pdf.

15. Shapiro-Mendoza CK, Lackritz EM. Epidemiology of late and moderate preterm birth.Semin Fetal Neonatal Med.2012; 17 (3): 120-5. 